\title{
Genetic trade-off between early fecundity and longevity in Bactrocera cucurbitae (Diptera: Tephritidae)
}

\author{
TAKAHISA MIYATAKE* \\ Okinawa Prefectural Agricultural Experiment Station, 4-222 Sakiyama-cho, Naha, Okinawa 903, Japan
}

\begin{abstract}
The response to selection on age at reproduction was measured to test for a genetic trade-off between early fecundity and longevity in the melon fly Bactrocera cucurbitae (Coquillett). Three replicate lines were selected for propagation by breeding from young (Y-lines) and old (O-lines) adults, respectively. Selection was continued for 24 and 9 generations for Y- and $\mathrm{O}$-lines, respectively. Females from O-lines lived longer than Y-line females as an indirect response to selection, indicating that longevity is a trait under genetic control. Females from Y-lines had higher fecundity early in the lifespan, a shorter preoviposition period, and a shorter prepeak fecundity period than females from O-lines. However, total fecundity did not differ between lines from the two selection regimes. These results suggest the existence of a genetic trade-off between early fecundity and longevity in the population examined, which may be controlled by pleiotropy. The larval period of the O-lines was longer than that of the Y-lines, whereas there were no significant effects of selection regime on egg hatchability or preadult survival rate. The trade-off relationship between early fecundity and longevity is discussed in relation to mass production of the melon fly for the sterile insect technique programme.
\end{abstract}

Keywords: Bactrocera cucurbitae, fecundity, longevity, pleiotropy, selection, trade-off.

\section{Introduction}

Since Haldane (1941), Medawar (1946, 1952), and Williams (1957) introduced evolutionary explanations for the evolution of ageing, a discussion of mechanisms has lasted for almost a half century (Rose, 1983, 1991; Rose \& Service, 1985; Partridge \& Barton, 1993; Zwaan et al., 1995). Theoretically, there are two explanations that are not mutually exclusive. The first is the antagonistic pleiotropy theory that was introduced by Medawar $(1946,1952)$ and Williams (1957), in which survival and late fecundity in life are sacrificed for the sake of early fecundity or fitness in the preadult period. This theory supposes that the alleles which increase fitness early in life, but have deleterious effects late in life, can be favoured by selection. This idea relates to a genetic trade-off between early fecundity and longevity. The second is the mutation accumulation theory, that was introduced by Medawar (1946, 1952), in which a life history might be depressed

*E-mail: qzg00607@niftyserve.or.jp below an optimal compromise by deleterious mutations, and ageing might evolve because of a greater mutation load on the later part of the life history.

A useful method for detecting genetic trade-offs is an artificial selection experiment for early and late reproduction (Rose \& Service, 1985; Reznick, 1992; Stearns, 1992; Partridge \& Barton, 1993; Nusbaum \& Rose, 1994). If antagonistic pleiotropy plays an important role in the evolution of ageing, a drop in early reproduction should be found if longevity increases. Lines selected for early reproduction should have high early fecundity with low longevity, whereas the lines selected for late reproduction should have high longevity with high late fecundity, and lower early fecundity.

Four artificial selection experiments testing for genetic trade-offs between early fecundity and longevity in Drosophila melanogaster, the geneticist's fruit fly, showed varying results. Two selection experiments found a genetic trade-off (Rose, 1984; Luckinbill et al., 1984), whereas the other two did not (Engström et al., 1992; Partridge \& Fowler, 1992). In a coleopterous species, Acanthoscelides 
obtectus, a genetic trade-off was not detected (Tucić et al., 1990; Gliksman \& Tucić, 1991). Although Rose and his colleague found a genetic trade-off in some selected lines in D. melanogaster (Rose, 1984; Hutchinson, 1989), it disappeared after long-term selection (Leroi et al., 1994a). Leroi et al. (1994a,b) suggested that the disappearance may have been caused by the following three reasons: (1) geneticby-environment interaction for life history traits; (2) the early fixation of segregating alleles at loci responsible for allocation; or (3) the appearance of epistatic alleles. Thus unsolved problems still remain in this area, even though many selection experiments have been conducted in $D$. melanogaster and other insect species.

Selection experiments for age of reproduction have been limited to $D$. melanogaster and a few coleopteran species (Mertz, 1975; Tucić et al., 1990; Gliksman \& Tucić, 1991). The accumulation and comparison of data from many taxa should be conducted to generalize the issue of the evolution of ageing. In this paper, an artificial selection experiment for early and late reproduction was conducted to detect heritable variation in longevity, and to test for a genetic trade-off between early fecundity and longevity in the melon fly Bactrocera cucurbitae (Coquillett). It should be noted that the selection was carried out on age at reproduction, and not longevity directly (see Zwaan et al., 1995). Age-specific fecundity, preoviposition period, prepeak fecundity period, total fecundity, larval period, egg hatchability, and preadult survival rate of the selected lines were also compared to detect correlated responses to selection. The results are also discussed in relation to the mass production of the melon fly for the sterile insect technique programme.

\section{Materials and methods}

\section{Selection lines}

The base population for this experiment was the mass-reared strain maintained for 63 generations in the Okinawa Prefectural Fruit Fly Eradication Project Office, Okinawa, Japan, according to the methods described by Nakamori \& Kakinohana (1980) and Nakamori et al. (1992). Kakinohana \& Yamagishi (1991) divided the mass-reared flies into two strains at 33 generations following introduction. In the first strain, flies were derived from eggs collected from the second to the sixth week after adult emergence (nonselected strain). In the second strain, flies were derived from eggs collected only from adults five to six weeks after eclosion (selected strain). The nonselected strain was used as the base population.

Three replicate 'young' and 'old' lines (total, six lines) were produced from the base population. Selection was started in June 1992, and was initiated from c. 3000 pupae of the mass-reared strain. These pupae were divided into three populations, and each placed in a rearing cage ( 40 by 30 by $28 \mathrm{~cm}$ ). The adults (c. 1000 flies) were allowed to eclose in each cage. When the age of these adults was $10-15$ and 55-60 days, eggs were collected from flies of each cage for $13 \mathrm{~h}$ (from 16.00 to 10.00 next morning) by using artificial oviposition cylinders (Sugimoto, 1978). These lines originating from the eggs collected at different ages were named young lines (Y-lines) and old lines (O-lines), respectively. About 2400 eggs $(0.3 \mathrm{~mL})$ were placed on $300 \mathrm{~g}$ of larval artificial medium (Nakamori et al., 1992) in a sample container ( $130 \mathrm{~mm}$ diameter, $77 \mathrm{~mm}$ height) for each line. Each cup was kept separately in a larger sample container $(150 \mathrm{~mm}$ diameter, $92 \mathrm{~mm}$ height) filled with water $(80 \mathrm{~mL})$. Water was exchanged for a pupation substrate consisting of a 7:1 mixture of sawdust and water at 4 days after egg seeding. Mature larvae jumped out from the medium and pupated in the pupation substrate. The pupae were put into a plastic cup $(200 \mathrm{~mL})$, and set in each rearing cage. These selection regimes were continued for 24 generations for Y-lines and 9 generations for $\mathrm{O}$-lines. Selection and all the experiments were conducted in a laboratory at $25 \pm 2^{\circ} \mathrm{C}$ under a photoperiod of 14:10 (L:D) h (light phase $05.30-19.30 \mathrm{~h}$ ).

\section{Longevity and fecundity of females from selected lines}

The longevity and fecundity of females from all lines were measured at generation 21 for $Y$ - and 8 for O-lines. Thirty one-day-old males and females that emerged on the same day were picked up randomly from each line and were paired. Each pair was put into an adult rearing cup (Miyatake, 1996a) to test the longevity of the melon fly (6 lines $\times 30$ cups $=180$ cups). A small piece of sliced pumpkin was placed at the bottom of the cup to stimulate oviposition at 3 days after eclosion according to the method described by Itô \& Yamagishi (1989). Eggs were collected every 7 days until the female died. The melon fly females used in this experiment did not lay eggs if a piece of host fruit or juice was not supplied. Eggs laid on the slice were collected with a fine brush the next morning (c. $18 \mathrm{~h}$ after oviposition) and placed on a Petri dish on a black board for 
counting. The diet and water were changed every week, and cups were exchanged at 3-week intervals to prevent mould growing on the excreta of the flies accumulated inside the cup. When a paired male died, another survivor male from the same line was put in the cup as soon as possible. Thus, the longevity and fecundity of the females examined may include the male effects, for example differences in fertility or sperm production, mating frequency, and locomotor activity, etc., because the males of these lines must also have responded to selection.

\section{Egg hatchability, preadult survival rate and larval period of selected lines}

Egg hatchability, preadult survival rate and larval period were measured at generation 24 for $\mathrm{Y}$ - and 9 for O-lines. Females of the six lines (Y-1-3, O-1-3) were allowed to lay eggs for $1 \mathrm{~h}$ (c. 20 days after emergence). These females (c. 500 flies) were exposed to c. 500 males of the same lines during about 20 days after emergence before measuring hatchability. Thus, male effects for egg hatchability can not be excluded. In the experiment for egg hatchability, 10 eggs collected from each line were placed on a piece of black gauze in a Petri dish. Ten Petri dishes were set for each of the six lines (10 eggs by 10 replicates $=100$ eggs for each line). The number of hatched eggs was counted after more than $30 \mathrm{~h}$, because the length of the egg stage of the melon fly at $27^{\circ} \mathrm{C}$ is about $25 \mathrm{~h}$ (Arakaki et al., 1984).

To examine larval period and preadult survival rate, 10 eggs collected from each line were placed on $15 \mathrm{~g}$ of the larval artificial medium in a small plastic cup $(20 \mathrm{~mL})$. Each cup was kept in a large plastic cup $(160 \mathrm{~mL})$ with water $(20 \mathrm{~mL})$. Mature larvae that 'popped' from the medium into the water were transferred to another plastic cup $(120 \mathrm{~mL})$ containing the pupation substrate. The number of mature larvae in the water for each cup was counted every day in the afternoon, because most of the 'popping' behaviour of the melon fly occurred in the morning (Miyatake, 1993). The period from egg seeding to collection of larvae in the water was defined as the larval period. Preadult survival rates were obtained as ratios of the number of emerged adults to the number of eggs seeded in each cup.

\section{Data analysis}

The Log Rank test, a distribution-free method, was used for statistical analysis of longevity between selected lines for each replicate, because longevity was not normally distributed (Lee, 1992). An unbalanced one-way ANOVA on selection regime, with replicate lines nested, was used to compare the age-specific fecundity between the $\mathrm{Y}$ - and $\mathrm{O}$-lines in each sampling interval (Sokal \& Rohlf, 1981, p. 294). A nested ANOVA was used for the analysis of the other correlated traits, i.e. preoviposition period, prepeak fecundity period (period before peak fecundity), total fecundity, larval period, egg hatchability and preadult survival rate. To normalize the data, a $\log$ transformation was used for the preoviposition period, prepeak fecundity period, total fecundity and larval period. An arcsine square root transformation was used for egg hatchability and preadult survival rate.

\section{Results}

\section{Longevity and fecundity of females from selected lines}

The survivorship curves for females of all lines are shown in Fig. 1. Females of the Y-lines had significantly $(P<0.0001)$ lower longevity than those from $\mathrm{O}$-lines. The average percentage of the total number of eggs laid by each female surviving in each week for each line is given in Fig. 2. Females of the $Y$-lines laid significantly more eggs than the O-females in week $1\left(F_{1,4}=63.06, P<0.001\right)$, week 2 $\left(F_{1,4}=21.30, \quad P<0.001\right)$, week $3 \quad\left(F_{1,4}=50.71\right.$, $P<0.001)$ and week $15 \quad\left(F_{1,4}=13.13, P<0.05\right)$. Females of the O-lines laid eggs continuously after all of the Y-line females died (Fig. 2). There were significant differences between replicate lines within a selection regime in week $8\left(F_{4,139}=4.63, P<0.001\right)$, week $9 \quad\left(F_{4,131}=4.55, \quad P<0.001\right)$ and week 10 $\left(F_{4,118}=5.42, P<0.0001\right)$.

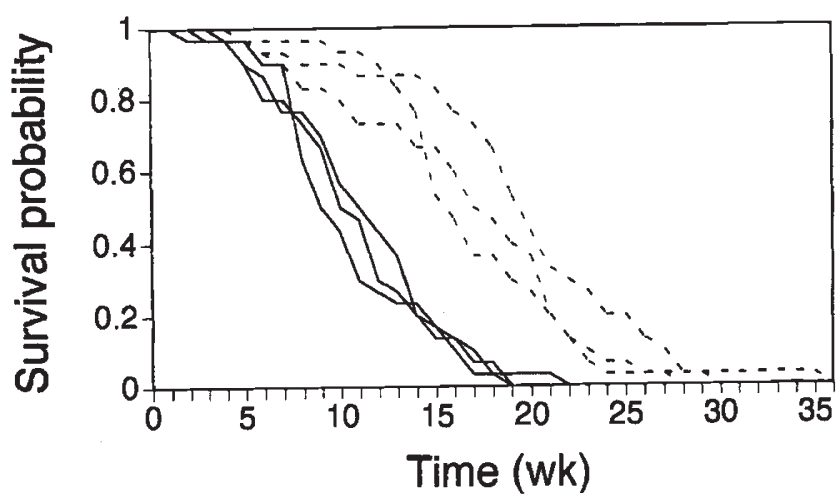

Fig. 1 Proportion of females surviving each week (cumulative survival probability) for selected lines. Solid lines represent the $\mathrm{Y}$-lines and dashed lines the O-lines. 
Comparisons of preoviposition period, prepeak fecundity period, and total fecundity per life among selected lines suggested that there was an association between selection regime and the first two traits, but no association between selection regime and total fecundity per life (Tables 1 and 2). Females of the Y-lines had significantly shorter preoviposition and prepeak fecundity periods than those of the O-lines (Table 2).

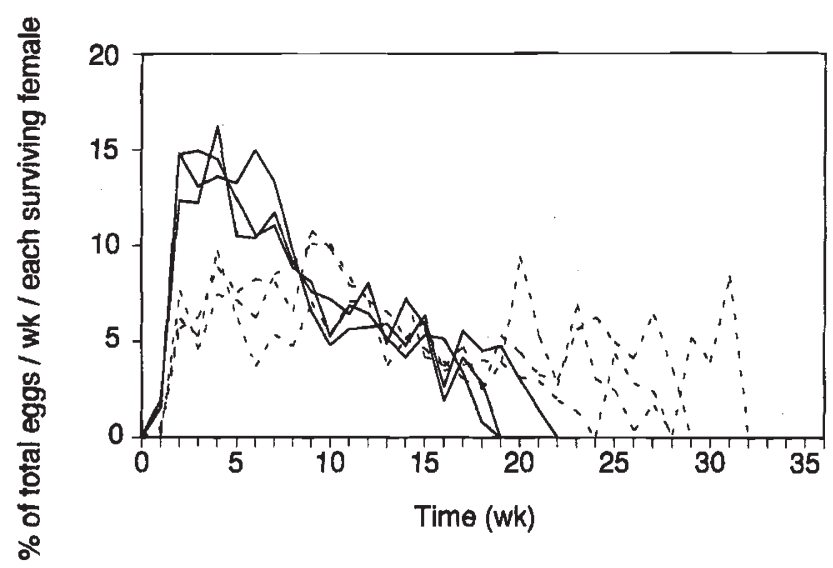

Fig. 2 Average percentage of the total number of eggs laid by each female surviving per week for selected lines. Solid lines represent the Y-lines and dashed lines the O-lines.

\section{Egg hatchability, preadult survival rate and larval period of selected lines}

Comparisons of egg hatchability, preadult survival rate and larval period among selected lines suggested that there was an association between selection regime and larval period, but no association between selection regime and the other two traits (Tables 3 and 4). Flies from the Y-lines had significantly shorter larval period than those from the O-lines (Table 4).

\section{Discussion}

An indirect response to selection for longevity, i.e. the O-line flies lived longer than those from the Y-lines, was shown in the mass-reared $B$. cucurbitae (Fig. 1). In $D$. melanogaster, almost all populations that were selected for older age at reproduction showed increased longevity as indirect responses to selection (Rose \& Charlesworth, 1981b; Rose, 1984; Luckinbill et al., 1984; Engström et al., 1992; Partridge \& Fowler, 1992) except in a few cases (Lints \& Hoste, 1977). Indirect responses to selection for longevity were also shown in some Coleoptera (Mertz, 1975; Tucić et al., 1990; Gliksman \& Tucić, 1991).

A genetic trade-off between early fecundity and longevity was clearly found in the current study (Fig. 2). It suggests that the mass-reared population had a negative genetic correlation between early

Table 1 Mean (and standard deviation) preoviposition period (wk), prepeak fecundity period and total fecundity per life of selected lines of the melon fly, Bactrocera cucurbitae

\begin{tabular}{lccc}
\hline & & \multicolumn{2}{c}{ Selection regime } \\
\cline { 3 - 4 } Traits & Replicate & Young & Old \\
\hline Preoviposition period (wk) & 1 & $1.8(0.9)$ & $2.5(1.1)$ \\
& 2 & $2.2(1.0)$ & $2.6(1.1)$ \\
& 3 & $2.2(1.0)$ & $3.3(2.6)$ \\
Prepeak fecundity period (wk) & 1 & $2.0(1.0)$ & $2.8(1.8)$ \\
& 2 & $4.8(2.6)$ & $7.3(4.0)$ \\
& 3 & $4.7(2.8)$ & $8.3(3.7)$ \\
Total fecundity per life & Mean & $4.6(2.8)$ & $10.7(5.7)$ \\
& 1 & $506.4(308.4)$ & $778.0(299.8)$ \\
& 2 & $465.5(245.8)$ & $556.7(357.1)$ \\
& 3 & $545.1(369.5)$ & $586.4(386.9)$ \\
& Mean & $505.7(313.7)$ & $640.4(363.3)$ \\
\hline
\end{tabular}

Standard deviations in parentheses. 
Table 2 Nested ANOVAS on preoviposition period, prepeak fecundity period and total fecundity per life of selected lines of the melon fly, Bactrocera cucurbitae

\begin{tabular}{llrrc}
\hline Traits & Source & SS & d.f. & $F$ \\
\hline Preoviposition period & Selection regime & 0.6934 & 1 & $12.70^{*}$ \\
& Replication & 0.2179 & 4 & 1.38 \\
& Error & 6.6442 & 168 & \\
Prepeak fecundity period & Selection regime & 3.1617 & 1 & $26.19^{* *}$ \\
& Replication & 0.4829 & 4 & 1.84 \\
& Error & 11.0210 & 168 & \\
Total fecundity per life & Selection regime & 0.7812 & 1 & 3.17 \\
& Replication & 0.9845 & 4 & 1.32 \\
& Error & 32.4761 & 174 & \\
\hline
\end{tabular}

Error term for selection regime is replication mean square.

${ }^{*} P<0.05,{ }^{* *} P<0.01$.

Table 3 Mean and standard deviation of egg hatchability (per cent), preadult survival rate (per cent) and larval period (days) of selected lines of the melon fly, Bactrocera cucurbitae

\begin{tabular}{lccc}
\hline & & \multicolumn{2}{c}{ Selection regime } \\
\cline { 3 - 4 } Traits & Replicate & Young & Old \\
\hline Egg hatchability (per cent) & 1 & $83.0(9.0)$ & $93.0(6.4)$ \\
& 2 & $84.0(9.0)$ & $89.0(8.3)$ \\
& 3 & $77.0(14.1)$ & $85.0(9.2)$ \\
Preadult survival rate (per cent) & 1 & $61.3(11.4)$ & $89.0(8.6)$ \\
& 2 & $76.5(16.3)$ & $78.3(14.6)$ \\
& 3 & $83.3(16.4)$ & $78.3(15.1)$ \\
Larval period (days) & Mean & $75.8(16.5)$ & $77.2(14.8)$ \\
& 1 & $6.39(0.29)$ & $6.88(0.35)$ \\
& 2 & $6.45(0.30)$ & $6.85(0.30)$ \\
& 3 & $6.60(0.40)$ & $6.95(0.28)$ \\
& Mean & $6.48(0.35)$ & $6.89(0.31)$ \\
\hline
\end{tabular}

Standard deviations in parentheses.

fecundity and longevity, which would indicate antagonistic pleiotropy. This correlation was also strongly supported by the following three points: (1) late fecundity in the O-lines after $\mathrm{Y}$-line females died out (Fig. 2); (2) the shorter preoviposition periods and shorter prepeak fecundity periods of the $\mathrm{Y}$-lines than those of the $\mathrm{O}$-lines (Tables 1 and 2); and (3) the same total fecundity per life in Y-and O-lines (Tables 1 and 2). These results also support the antagonistic pleiotropy theory in B. cucurbitae. This theory is supported by some selection experiments in D. melanogaster (Rose, 1984; Luckinbill et al., 1984), and also by experiments using classical quantitative genetic techniques to estimate genetic variance and covariance among fitness components at different ages (Rose \& Charlesworth, 1981a; Roach, 1986; Tucić et al., 1988; Tanaka, 1993). However, some studies on Drosophila using the classical technique do not support this theory (Giesel, 1986; Engström et al., 1989). More data are required in other taxonomic groups to evaluate the antagonistic pleiotropy theory. Tephritid flies, the so-called applied entomologist's fruit flies, which are well studied for their biology (Robinson \& Hooper, 1989), should be regarded as a candidate group for selection experiments and other forms of quanti- 
Table 4 Nested ANOVAS on egg hatchability, preadult survival rate (per cent) and larval period (days) of selected lines of the melon fly, Bactrocera cucurbitae

\begin{tabular}{llcrc}
\hline Traits & Source & SS & d.f. & $F$ \\
\hline Egg hatchability & Selection regime & 0.1106 & 1 & 5.23 \\
& Replication & 0.0846 & 4 & 1.65 \\
Preadult survival rate & Error & 0.6939 & 54 & \\
& Selection regime & 0.0014 & 1 & 0.04 \\
& Replication & 0.1423 & 4 & 1.44 \\
Larval period & Error & 1.6299 & 66 & \\
& Selection regime & 0.0131 & 1 & $36.67^{* *}$ \\
& Replication & 0.0014 & 4 & 0.75 \\
\hline
\end{tabular}

Error term for selection regime is replication mean square. ${ }^{* *} P<0.01$.

tative genetic analysis (McInnis, 1987; Wood \& Harris, 1989; Miyatake, 1995, 1996b).

The Y-lines had a shorter larval period than the O-lines in the current study (Tables 3 and 4). Partridge \& Fowler (1992) found a similar result in D. melanogaster. Roper et al. (1993) proposed two reasons for the shortened larval period in the old lines of Partridge \& Fowler (1992). The first was the different larval density between the two lines selected for early and late reproduction; the young lines were subjected to a higher rearing density than the old lines, then the larval period of the young lines was shortened. The second reason was that the flies which sexually matured early with short larval periods were favoured by the selection method for the young lines. Because in the current study the same numbers of eggs were seeded in both selected lines and no difference in the survival rate between both lines was observed, the first reason is unacceptable. The second reason could explain the difference of larval period. The mass-reared melon fly females mature at 9.1 \pm 3.9 days (mean \pm SD) (Kuba \& Soemori, 1988). If eggs were collected from 10 to 15-day-old adults, offspring of females that matured later would not be included in the following generations. This could cause inadvertent selection for rapid development in the females of the young lines (Roper et al., 1993).

In the current study, body size of selected lines was not measured. Miyatake (1995) showed that the lines selected for longer developmental period had larger body size than the lines selected for shorter developmental period in B. cucurbitae. However, longer developmental periods were not associated with higher fecundity and longevity (Miyatake, 1996a).
Lower longevity of the mass-reared strain than of wild flies has often been reported in B. cucurbitae (Soemori \& Nakamori, 1981; Kamikado et al., 1987; Kakinohana \& Yamagishi, 1991). As described above, Kakinohana \& Yamagishi (1991) divided the mass-reared flies of Okinawa into two strains at 33 generations; nonselected and selected strains. Survival rate at the tenth week in the selected strain was significantly greater than in the nonselected strain after 34 generations (Miyatake \& Yamagishi, 1993). This nonselected strain was used in the current study as the base population.

The current study revealed a genetic basis for the difference between the selected and nonselected lines in the practical mass selection of the melon fly. It seems worthwhile to examine whether O-lines have increased longevity compared to the massreared strain. The current study also suggests that the selected strain must have lower early fecundity than the nonselected strain in practical mass rearing. It would be worthwhile to examine the male mating ability of the selected lines as a correlated response to selection, because the sterile insect technique depends on the mating ability of the released males.

\section{Acknowledgements}

I am grateful to Linda Partridge (University College London) and Kenjiro Kawasaki (National Institute of Sericultural and Entomological Science, Japan) for critical reviews of the manuscript.

\section{References}

ARAKAKI, N., KUBA, H. AND SOEMORI, H. 1984. The effect of water storage temperature on the hatching rate of

(C) The Genetical Society of Great Britain, Heredity, 78, 93-100. 
melon fly, Dacus cucurbitae Coquillett, eggs. Bull. Okinawa Agric. Exp. Stn., 9, 113-118.

ENGSTRÖM, G., LILJEDAHL, L. E., RASMUSON, M. AND BJÖRKLUND, T. 1989. Expression of genetic and environmental variation during ageing. 1. Estimation of variance components for number of adult offspring in Drosophila melanogaster. Theor. Appl. Genet., 77, 119-122.

ENGSTRÖM, G., LILJEDAHL, L. E. AND BJÖRKLUND, T. 1992. Expression of genetic and environmental variation during ageing. 2. Selection for increased lifespan in Drosophila melanogaster. Theor. Appl. Genet., 85, 26-32.

GIESEL, J. T. 1986. Genetic correlation of life history variables in outbred, wild Drosophila melanogaster: effects of photoperiod regimen. Am. Nat., 128, 593-603.

GLIKSMAN, 1. AND TUCIĆ, N. 1991. Effects of selection for early and late reproduction in low and high larval density populations of the bean weevil (Acanthoscelides obtectus). Génét. Sél. Évol., 23, 119-132.

haldane, J. B. s. 1941. New Paths in Genetics. Allen and Unwin, London.

Hutchinson, E. w. 1989. Quantitative Genetics of Postponed Senescence. Ph. D. Thesis, Dalhousie University, Halifax.

ITÔ, Y. AND YAMAGISHI, M. 1989. Sperm competition in the melon fly, Dacus cucurbitae (Diptera: Tephritidae): effects of sequential matings with normal and virgin or non-virgin sterile males. Appl. Ent. Zool., 24, 466-477.

KAKINOHANA, H. AND YAMAGISHl, M. 1991. The mass production of the melon fly techniques and problems. In: Kawasaki, K., Iwahashi, O. and Kaneshiro, K. (eds) Biology and Control of Fruit Flies (Proc. Int. Symp. Okinawa, Japan, 1991), pp. 1-10. Ginowan, Okinawa, Japan.

KAMIKADO, T., CHISHAKI, N., KAMIWADA, H. AND TANAKA, A. 1987. Mass rearing of the melon fly, Dacus cucurbitae Coquillett, by the sterile insect release method. I. Changes in the amount of eggs laid and longevity of mass-reared adults. Proc. Ass. Pl. Prot. Kyushu, 33, 164-166 (in Japanese with English summary).

KUBA, H. AND SOEMORI, H. 1988. Characteristics of copulation duration, hatchability of eggs and remating intervals in the melon fly, Dacus cucurbitae Coquillett (Diptera: Tephritidae). Jap. J. Appl. Ent. Zool., 32, 321-324 (in Japanese with English summary).

LEE, E. T. 1992. Statistical Methods for Survival Data Analysis, 2nd edn. John Wiley and Sons, New York.

LEROI, A. M., CHIPPINDAle, A. K. AND ROSE, M. R. 1994a. Long-term laboratory evolution of a genetic life-history trade-off in Drosophila melanogaster. 1. The role of genotype-by-environment interaction. Evolution, 48, 1244-1257.

LEROI, A. M., CHEN, W. R. AND ROSE, M. R. 1994b. Longterm laboratory evolution of a genetic life-history tradeoff in Drosophila melanogaster. 2. Stability of genetic correlations. Evolution, 48, 1258-1268.

LINTS, F. A. AND HOSTE, c. 1977. The Lansing effect revisited. II. - Cumulative and spontaneously reversible parental age effects on fecundity in Drosophila melano- gaster. Evolution, 31, 387-404.

LUCKINBILL, L. S., ARKING, R., CLARE, M. J., CIROCCO, W. C. AND BUCK, s. A. 1984. Selection for delayed senescence in Drosophila melanogaster. Evolution, 38, 996-1003.

MclNNIS, D. O. 1987. Mediterranean fruit fly (Diptera: Tephritidae): directional selection for large and small pupal size. Ann. Entomol. Soc. Am., 80, 333-338.

MEDAWAR, P. B. 1946. Old age and natural death. Modern Quart., 1, 30-56.

medawar, P. B. 1952. An Unsolved Problem of Biology. H. K. Lewis, London.

MERTZ, D. R. 1975. Senescent decline in flour beetle strains selected for early adult fitness. Physiol. Zool., 48, $1-23$.

MIYATAKE, T. 1993. Difference in the larval and pupal periods between mass-reared and wild strains of the melon fly, Bactrocera cucurbitae (Coquillett) (Diptera: Tephritidae). Appl. Ent. Zool., 28, 577-581.

MIYATAKE, T. 1995. Two-way artificial selection for developmental period in Bactrocera cucurbitae (Diptera: Tephritidae). Ann. Entomol. Soc. Am., 88, 848-855.

MIYATAKE, T. 1996a. Comparison of adult life history traits in lines artificially selected for long and short larval and pupal developmental periods in the melon fly, Bactrocera cucurbitae (Diptera: Tephritidae). Appl. Ent. Zool., 31, 335-343.

miyatake, T. 1996b. Artificial selection experiments in the melon fly: the status quo and problems. In: McPheron, B. A. and Steck, G. J. (eds) Fruit Fly Pests: A World Assessment of Their Biology and Management, pp. 437-443. St. Lucie Press, Delray Beach, FL.

miYATAKE, T. AND YAMAGishl, M. 1993. Active quality control in mass reared melon flies: quantitative genetic aspects. In: Management of Insect Pests: Nuclear and Related Molecular Genetic Techniques, pp. 201-213. IAEA, Vienna.

NAKAMORI, H. AND KAKINOHANA, H. 1980. Mass-production of the melon fly, Dacus cucurbitae Coquillett, in Okinawa, Japan. Rev. Plant Prot. Res., 13, 37-53.

NAKAMORI, H., KAKINOHANA, H. AND YAMAGISHI, M. 1992. Automated mass production system for fruit flies based on the melon fly, Dacus cucurbitae Coquillett (Diptera: Tephritidae). In: Anderson, T. E. and Leppla, N. C. (eds) Advances in Insect Rearing for Research and Pest Management, pp. 441-454. Westview Press, Boulder, CO.

nusbaum, T. J. ANd Rose., M. R. 1994. Aging in Drosophila. Comp. Biochem. Physiol., 109, 33-38.

PARTRIDGE, L. AND BARTON, N. H. 1993. Optimality, mutation and the evolution of ageing. Nature, 362, 305-311.

PARTRIDGE, L. AND FOWLER, K. 1992. Direct and correlated responses to selection on age at reproduction in Drosophila melanogaster. Evolution, 46, 76-91.

REZNICK, D. 1992. Measuring the costs of reproduction. Trends Ecol. Evol., 7, 42-45.

ROACH, A. D. 1986. Life history variation in Geranium carolinianum. 1. Covariation between characters at different stages of the life cycle. Am. Nat., 128, 47-57.

ROBINSON, A. S. AND HOOPER, G. 1989. Fruit Flies: Their

(c) The Genetical Society of Great Britain, Heredity, 78, 93-100. 
Biology, Natural Enemies and Control, vols $3 \mathrm{~A}$ and B. Elsevier, Amsterdam.

ROPER, C., PIGNATElli, P. AND PARTRIDGE, L. 1993. Evolutionary effects of selection on age at reproduction in larval and adult Drosophila melanogaster. Evolution, 47, $445-455$.

Rose, M. R. 1983. Theories of life-history evolution. Am. Zool., 23, 15-23.

ROSE, M. R. 1984. Artificial selection on a fitness-component in Drosophila melanogaster. Evolution, 38, 516-526.

ROSE, M. R. 1991. Evolutionary Biology of Aging. Oxford University Press, Oxford.

ROSE, M. R. AND CHARLESWORTH, B. 1981a. Genetics of life history in Drosophila melanogaster. I. Sib analysis of adult females. Genetics, 97, 173-186.

ROSE, M. R. AND CHARLESWORTH, B. 1981b. Genetics of life history in Drosophila melanogaster. II. Exploratory selection experiments. Genetics, 97, 187-196.

ROSE, M. R. AND SERVICE, P. M. 1985. Evolution of aging. Rev. Biol. Res. Aging, 2, 85-98.

SOEMORI, H. AND NAKAMORI, H. 1981. Production of successive generations of a new strain of the melon fly, Dacus cucurbitae Coquillett (Diptera: Tephritidae) and reproductive characteristics in mass rearing. Jap. J. Appl. Ent. Zool., 25, 229-235 (in Japanese with an English summary).

SOKAL, R. R. AND ROHLF, F. J. 1981. Biometry, 2nd edn.
Freeman, New York.

STEARns, s. C. 1992. The Evolution of Life Histories. Oxford University Press, Oxford.

sugimoto, A. 1978. Egg collection method in mass rearing of the melon fly, Dacus cucurbitae Coquillett (Diptera: Tephritidae). Jap. J. Appl. Ent. Zool., 22, 60-67 (in Japanese with English summary).

TANAKA, Y. 1993. A genetic mechanism for the evolution of senescence in Callosobruchus chinensis (the azuki bean weevil). Heredity, 70, 318-321.

TUCiĆ, N., CVETKOVIĆ, D. AND Milanović, D. 1988. The genetic variation and covariation among fitness components in Drosophila melanogaster females and males. Heredity, 60, 55-60.

TUCIĆ, N., CVETKOVIĆ, D., STOJILJKOVIĆ, V. AND BEJAKOVIĆ, D. 1990. The effects of selection for early and late reproduction on fecundity and longevity in bean weevil (Acanthoscelides obtectus). Genetica, 80, 221-227.

WILliams, G. C. 1957. Pleiotropy, natural selection, and the evolution of senescence. Evolution, 11, 398-411.

WOOD, R. J. AND HARRIS, D. J. 1989. Artificial and natural selection. In: Robinson, A. S. and Hooper, G. (eds) Fruit Flies, Their Biology, Natural Enemies and Control, Vol. 3B, pp. 18-31. Elsevier, Amsterdam.

ZWAAN, B., BIJLSMA, B. AND HOEKSTRA, R. F. 1995. Direct selection on life span in Drosophila melanogaster. Evolution, 49, 649-659. 